\title{
Influencia estacional en las características de los pacientes ingresados en una unidad de cuidados intensivos
}

\author{
L. Santana Cabrera*, M. Sánchez-Palacios, A. Uriarte Rodriguez, M. Fernández Arroyo, \\ S. Martínez Cuéllar y R. Lorenzo Torrent
}

Servicio de Medicina Intensiva, Hospital Universitario Insular de Gran Canaria, Gran Canaria, España

Recibido el 26 de mayo de 2009; aceptado el 10 de julio de 2009

Disponible en Internet el 25 de septiembre de 2009

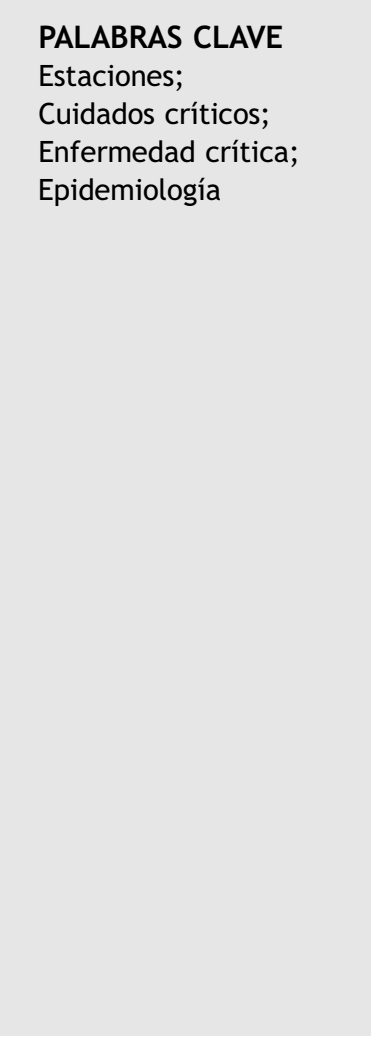

\begin{abstract}
Resumen
Objetivo: Estudiar la variabilidad estacional en la epidemiología y el pronóstico de la enfermedad crítica.

Diseño: Estudio retrospectivo de datos recogidos prospectivamente durante el período 2001 a 2008.

Ámbito: Unidad de Cuidados Intensivos (UCI) polivalente del Hospital Universitario Insular de Gran Canaria.

Pacientes: Todos los pacientes que ingresaron en la $\mathrm{UCl}$, procedentes del Servicio de Urgencias, según el período estacional (primavera, verano, otoño e invierno).

Variables de interés principales: Se recogieron datos demográficos y diagnósticos de los pacientes al ingreso, Acute Physiology and Chronic Health Evaluation [APACHE II], la necesidad de ventilación mecánica y, por último, la mortalidad.

Resultados: Durante el período de estudio ingresaron en nuestra $\mathrm{UCI}$, procedentes del Servicio de Urgencias, 3.115 pacientes, un $21 \%$ en verano, incidencia significativamente menor que en las otras estaciones del año $(p<0,001)$. No encontramos diferencias estadísticamente significativas entre los 4 grupos en relación con la edad, el tipo de pacientes, la gravedad al ingreso según APACHE II, ni en la mortalidad en la UCl. Sin embargo, por sexos sí encontramos diferencias significativas, ya que ingresan un mayor procentaje de mujeres en verano que en invierno (un $33 \%$ versus un $27 \%$; $p=0,037$ ). La estancia media en $\mathrm{UCl}$ de los que ingresaron en verano fue similar a los ingresados durante el invierno ( 4,9 días versus 5,8 días; $p=0,052$ ). La necesidad de ventilación mecánica y los días que precisaron de ésta, por grupos diagnósticos, fue similar entre el verano y el invierno. El análisis multivariado no mostró variables independientes asociadas al período estacional en el que ingresaron.

Conclusiones: La estabilidad climática de nuestro entorno implica que este factor no influya en el pronóstico de los pacientes que ingresan con enfermedad crítica.

(c) 2009 Elsevier España, S.L. y SEMICYUC. Todos los derechos reservados.
\end{abstract}

*Autor para correspondencia.

Correo electrónico: Isancabx@gobiernodecanarias.org (L. Santana Cabrera). 


\section{KEYWORD}

Seasons;

Critical care;

Critical illness;

Epidemiology

\section{Seasonal influence in characteristics of patients admitted to an intensive care unit}

\begin{abstract}
Objective: To investigate seasonal variability in the epidemiology and the outcome of critical illness.

Design: Retrospective analysis of prospectively collected data during the period 2001-2008. Setting: Polyvalent intensive care unit (ICU) of a tertiary hospital in the Canary Islands. Patients: Adult patients who were hospitalized in the ICU from the Emergency Department, according to the season period (spring, summer, autumn and winter).

Primary variables of interest: Demographic data, clinical diagnosis on ICU admission, APACHE II, need of mechanical ventilation and, finally, the mortality were collected.

Results: During the study period, 3,115 patients, coming from the Emergency Department, were hospitalized in our ICU. Of these, $21 \%$ were admitted during the summer, an incidence rate that is statistically lower than in other seasons of the year $(P<0.001)$. We did not find any statistically significant differences between the four groups according to the age, type of patients, severity at the moment of admission to the ICU, according to the APACHE II score or in the mortality rate in the ICU. However, significant differences were found in regards to gender. Admission of women during the summer was higher than in winter (33\% vs $27 \%, P=0.037)$. Average stay in the ICU of patients admitted in the summer was similar to winter ( 4.9 days vs 5.8 days; $P=0.052$ ). Need for mechanical ventilation and days it was required, by diagnostic groups, were similar between the summer and the winter. The multivariate analysis did not show independent variables associated with the seasonal period in which the patients were admitted.

Conclusions: The stability of our climate implies that this factor does not influence the prognosis of patients who are admitted with critical illness.

(c) 2009 Elsevier España, S.L. and SEMICYUC. All rights reserved.
\end{abstract}

\section{Introducción}

El pronóstico del paciente que ingresa en una Unidad de Cuidados Intensivos (UCI) depende, sin duda alguna, de la gravedad de la enfermedad crítica. Sin embargo, hay diferentes factores que se han relacionado con este pronóstico, con datos no concluyentes en la literatura médica, como son el sexo, la edad, la estancia prolongada, etc ${ }^{1,2}$.

Este pronóstico se ha relacionado también con factores externos, como la climatología, que se traduce en un incremento en el número de ingresos y un peor pronóstico de los pacientes que ingresan durante la estación invernal ${ }^{3-5}$.

Nosotros trabajamos en un entorno con una climatología que tiene una oscilación térmica de $6{ }^{\circ} \mathrm{C}$ entre las diversas estaciones, con una media entre 18 y $24{ }^{\circ} \mathrm{C}\left(64,4\right.$ y $\left.75,2{ }^{\circ} \mathrm{F}\right)$. Estamos situados a $4^{\circ}$ del trópico de Cáncer, paralelo que pasa por La Habana, próximos a la costa africana y bañados por los vientos alisios, lo que hace que nos encontremos en un clima subtropical. Por esto nos planteamos investigar si, al disponer de un clima uniforme durante todo el año, nuestros pacientes se ven libres o no de la influencia estacional en el pronóstico de la enfermedad grave. Por tanto, el objetivo de nuestro estudio era analizar la influencia de la variabilidad estacional en la epidemiología y el pronóstico de la enfermedad crítica.

\section{Pacientes y método}

Se trata de un estudio retrospectivo observacional de los datos recogidos prospectivamente sobre los pacientes procedentes del Servicio de Urgencias que ingresaron en nuestra $\mathrm{UCl}$, de carácter polivalente, de adultos y con una capacidad de 24 camas, de un hospital terciario. Se incluyeron todos los pacientes ingresados directamente desde urgencias durante el período comprendido entre enero de 2001 y diciembre de 2008, y se excluyeron los que procedían de las plantas de hospitalización o desde otros hospitales. Se analizaron variables demográficas, como la edad, el sexo, el tipo de paciente (coronario, médico, quirúrgico o traumatológico), el APACHE II al ingreso, los días de estancia en la $\mathrm{UCl}$, la necesidad de técnicas como la ventilación mecánica $y$, por último, la mortalidad en la UCl.

Se analizaron todos los pacientes ingresados durante el período estudiado y se compararon según el período estacional del año en el que ingresaban (primavera, verano, otoño e invierno), y según el grupo diagnóstico al que pertenecían (coronarios, médicos, quirúrgicos y traumatológicos), mediante las pruebas de la t de Student o la de Mann-Whitney para comparar variables continuas y la prueba de la $\chi^{2}$ o la de Fisher para comparar porcentajes, y se consideró significativa una $\mathrm{p}$ inferior a 0,05 . Los datos se expresan como media \pm desviación estándar (DE). Asimismo, se estudió la relación del período estacional con diversas variables potencialmente asociadas a ésta mediante el análisis de regresión logística multivariable, se utilizó el test de regresión logística binaria y se expresaron los resultados como odds ratio e intervalo de confianza del $95 \%$, y se consideró significativa una $\mathrm{p}$ inferior o igual a 0,05.

\section{Resultados}

Durante el período de estudio ingresaron en nuestra $\mathrm{UCl}$, procedentes del Servicio de Urgencias, 3.115 pacientes, de 
Tabla 1 Características de los pacientes ingresados según el período estacional $(n=3.115)$

\begin{tabular}{lllll}
\hline & Primavera & Verano & Otoño & Invierno \\
\hline $\mathrm{n}(\%)$ & $851(27,31)$ & $667(21,41)$ & $813(26,09)$ & $784(25,16)$ \\
Edad, años (media $\pm \mathrm{DE})$ & $55,53 \pm 16,31$ & $54,88 \pm 16,55$ & $55,54 \pm 15,62$ & $55,76 \pm 16,38$ \\
Sexo femenino, $\mathrm{n}(\%)$ & $255(29,96)$ & $220(33,08)$ & $241(29,62)$ & $219(27,93)$ \\
APACHE II & $10,74 \pm 7,7$ & $10,94 \pm 8,3$ & $10,61 \pm 7,9$ & $11,25 \pm 8$ \\
Grupos diagnósticos, $n(\%)$ & & & & \\
Coronarios & $414(48,70)$ & $303(45,42)$ & $418(51,41)$ & $371(47,32)$ \\
Médicos & $302(35,49)$ & $252(37,78)$ & $255(31,36)$ & $263(33,54)$ \\
Quirúrgicos & $69(8,1)$ & $55(8,2)$ & $72(8,9)$ & $72(9,2)$ \\
Traumas & $65(7,6)$ & $57(8,5)$ & $68(8,3)$ & $78(9,9)$ \\
Estancia media en UCl (días) & $5,7 \pm 9,1$ & $4,9 \pm 8,7$ & $5,3 \pm 9,2$ & $5,8 \pm 8,9$ \\
Necesidad de VM, $\mathrm{n}(\%)$ & $354(41,59)$ & $274(41,08)$ & $374(46)$ & $365(46,55)$ \\
Días de VM (media \pm DE) & $8,6 \pm 9,94$ & $7,3 \pm 11,23$ & $7,2 \pm 11,4$ & $8,1 \pm 9,7$ \\
Mortalidad UCI, $\mathrm{n}(\%)$ & $150(17,64)$ & $127(19,06)$ & $140(17,2)$ & $141(17,9)$
\end{tabular}

APACHE II:Acute Physiology and Chronic Health Evaluation; DE: desviación estándar; UCl: unidad de cuidados intensivos; VM: ventilación mecánica.

Tabla 2 Mortalidad según grupos diagnósticos en cada estación

\begin{tabular}{lllll}
\hline & Primavera & Verano & Otoño & Invierno \\
\cline { 2 - 5 } & $\mathrm{n}=150$ & $\mathrm{n}=127$ & $\mathrm{n}=140$ & $\mathrm{n}=141$ \\
\hline Coronarios, $\mathrm{n}(\%)$ & $28(18,6)$ & $20(15,7)$ & $34(24,3)$ & $20(14,2)$ \\
Médicos, $\mathrm{n}(\%)$ & $84(56)$ & $74(58,2)$ & $66(47,1)$ & $81(57,4)$ \\
Quirúrgicos, $\mathrm{n}(\%)$ & $20(13,3)$ & $19(14,9)$ & $21(15)$ & $24(17)$ \\
Traumas, $\mathrm{n}(\%)$ & $15(10)$ & $13(10,2)$ & $18(12,8)$ & $15(10,6)$ \\
\hline
\end{tabular}

los que $851(27,31 \%)$ ingresaron en primavera, $667(21,41 \%)$ en verano, $813(26,09 \%)$ en otoño y $784(25,16 \%)$ en invierno. Las características demográficas de los pacientes ingresados según la estación del año en la que ingresaran se pueden observar en la tabla 1. Al analizar las diferencias entre los pacientes según la estación del año en la que se los admitió en la $\mathrm{UCl}$, no encontramos diferencias estadísticamente significativas entre los 4 grupos en relación con la edad, el tipo de paciente, la gravedad al ingreso según APACHE II, ni en la mortalidad en la UCI.

Tampoco la mortalidad mostró diferencias significativas entre el verano y el invierno cuando analizamos a los pacientes según el grupo diagnóstico al que pertenecían (tabla 2).

En el total de la muestra, el sexo predominante de los pacientes que ingresan es el masculino; sin embargo, observamos una mayor proporción de mujeres admitidas en verano que en invierno (un $33 \%$ versus un $27 \%$; $p=0,037$ ).

También encontramos una diferencia casi significativa entre la estancia media en la $\mathrm{UCl}$ de los pacientes que ingresaron en verano, respecto al invierno; así, en verano era de 4,9 días mientras que en invierno de 5,8 días $(p=0,052)$.

En cuanto a la necesidad global de ventilación mecánica de los pacientes, fue mayor en invierno que en verano (un $46 \%$ versus un $41 \%$; $p=0,035$ ) y que en primavera (un $46 \%$ versus un $41 \%$; $p=0,043$ ), aunque no encontramos diferencias
Tabla 3 Análisis multivariable que estudia la influencia de las estaciones con diversos factores epidemiológicos

\begin{tabular}{llll}
\hline & OR & IC del 95\% & $\mathbf{p}$ \\
\hline Edad & 0,99 & $0,99-1$ & 0,85 \\
Sexo & 0,96 & $0,74-1,25$ & 0,80 \\
APACHE II & 0,98 & $0,97-1$ & 0,26 \\
Estancia media UCI & 1,02 & $0,99-1,04$ & 0,13 \\
Días de VM & 0,98 & $0,95-1,01$ & 0,44 \\
Tipo de paciente & 0,95 & $0,83-1,1$ & 0,56 \\
Mortalidad & 1,13 & $0,84-1,52$ & 0,39 \\
\hline
\end{tabular}

APACHE II:Acute Physiology and Chronic Health Evaluation; IC: intervalo de confianza; OR: odds ratio; UCI: unidad de cuidados intensivos; VM: ventilación mecánica.

entre las diferentes estaciones en cuanto a los días que precisaron de ésta.

Sin embargo, cuando analizamos a los pacientes que recibieron ventilación mecánica, según los grupos diagnósticos a los que pertenecían, no encontramos diferencias estadísticamente significativas entre el verano y el invierno.

En el análisis multivariable (tabla 3) no se encontró relación del período estacional con diversas variables 
que podrían estar potencialmente asociadas con ésta, tales como la edad, el sexo, el APACHE II, la estancia, los días de ventilación mecánica, el tipo de paciente o la mortalidad.

\section{Discusión}

Se ha estudiado la influencia del clima en la enfermedad y se ha demostrado que la exposición a un ambiente frío puede provocar trastornos orgánicos que pueden llevar, incluso, a la muerte. Se conoce la mayor propensión a la trombosis arterial producida por la hemoconcentración inducida por el frío intenso, también que puede provocar hipertensión, que puede llevar a la ruptura de placas de ateroma y, por último, producir un vasoespasmo coronario ${ }^{6}$. También es probable que la inmunosupresión debida a hormonas liberadas durante el estrés provocado por la exposición al frío disminuya la resistencia a las infecciones respiratorias; lo que, sumado a la broncoconstricción que produce el efecto directo del frío en la vía aérea, hace que se puedan incrementar los ingresos de pacientes con enfermedades respiratorias durante el invierno ${ }^{7}$.

Esto se ha corroborado con diferentes estudios que demuestran una mayor incidencia de enfermedades graves durante las estaciones invernales ${ }^{8}$, donde la incidencia de sepsis de origen respiratorio, debida a los cambios estacionales, variará de acuerdo a la zona geográfica ${ }^{9,10}$; así, por ejemplo, las infecciones neumocócicas se incrementan cada invierno, un fenómeno que no se ha explicado bien ${ }^{11}$. Otras enfermedades respiratorias, como la EPOC o el asma también tienen, en algunas series, un predominio de mortalidad en los meses de diciembre y enero $^{12,13}$.

Pero no sólo se ha encontrado relación estacional con enfermedades infecciosas; así, en Europa central se encuentra una elevada mortalidad de causa cardiovascular, sobre todo en hombres entre 25 y 59 años, que se podría atribuir a la exposición prolongada al frío durante el trabajo en época de invierno ${ }^{14,15}$. También se ha relacionado el incremento de la mortalidad por accidente cerebrovascular durante el invierno, sobre todo cuando se asocia a complicaciones respiratorias $^{16}$.

Apoya a esta teoría de la influencia del frío en la salud, el hecho de que se ha demostrado que tomar medidas protectoras contra éste, como vivir en viviendas más cálidas, ir más abrigado y ser más activo cuando se está al aire libre, parece disminuir la gravedad de estas enfermedades $^{17}$.

Habría que aclarar que la creencia de que el frío incrementa la mortalidad de algunas enfermedades no es del todo correcta, ya que lo que aumenta es la incidencia de algunas enfermedades que podrían resultar en un incremento en la mortalidad.

En la literatura médica revisada no hemos encontrado otros artículos realizados en regiones con una estabilidad climática durante todo el año, como la nuestra, que estudien cómo influye el clima en la epidemiología de la enfermedad crítica. Por este motivo, ya que nosotros habitamos en un clima cálido durante todo el año, por lo que se llama "islas afortunadas" a Canarias, nos decidimos a estudiar este aspecto.
El clima dominante en Canarias es el tropical seco y húmedo pero, debido a su posición en medio del Atlántico y a su relieve existen numerosos topoclimas muy significativos, predominantemente bajo el dominio de los vientos alisios procedentes del flanco oriental del anticiclón de las Azores. La combinación de los vientos y la corriente marina hace que las temperaturas en Canarias sean notablemente estables y menos calurosas de lo que por su latitud les correspondería. Las temperaturas más altas se alcanzan en agosto, aunque septiembre tiene prácticamente la misma media. Las temperaturas más frías se dan en enero, seguidas muy de cerca por las de febrero. La oscilación térmica entre el mes más cálido y el más frío está por debajo de los $10^{\circ} \mathrm{C}$, entre los $18{ }^{\circ} \mathrm{C}$ y los $24^{\circ} \mathrm{C}$, salvo en las cumbres montañosas que puede llegar a los $13{ }^{\circ} \mathrm{C}$.

Por la estabilidad climática en nuestro entorno, durante todo el año, encontramos que prácticamente no existen diferencias en el número de pacientes que ingresan por enfermedad crítica en nuestra $\mathrm{UCl}$ en las estaciones del año, salvo en el período de verano, durante el que ingresan menos pacientes, lo que podría ser atribuible al descenso en este período en la afluencia de turistas extranjeros, los que durante las demás estaciones ocupan un porcentaje significativo de ingresos.

Las características de los pacientes no difieren en las distintas estaciones, y eso, asociado a una mortalidad baja como corresponde a un APACHE medio relativamente bajo, hace que no haya diferencias de mortalidad estacional. La cuestión de si la estabilidad climática influye de igual manera en pacientes más graves habría que estudiarla en estudios posteriores.

Aunque encontramos, de forma global, un mayor número de pacientes que precisaban de ventilación mecánica en invierno, al analizarlos por grupos diagnósticos (coronarios, médicos, quirúrgicos y traumatológicos) no encontramos diferencias significativas en la necesidad de ventilación mecánica entre las estaciones de mayor y menor temperatura.

En conclusión, podríamos afirmar que la estabilidad climática de nuestro entorno implica que este factor no influya en el pronóstico de los pacientes que ingresan con enfermedad crítica, y que habría que completar con estudios posteriores en pacientes con mayor APACHE II al ingreso.

\section{Bibliografía}

1. Santana Cabrera L, Sánchez-Palacios M, Hernández Medina E, Eugenio Robaina P, Villanueva Hernández E. Características y pronóstico de los pacientes mayores con estancia muy prolongada en una Unidad de Cuidados Intensivos. Med Intensiva. 2008;32:157-62.

2. Santana Cabrera L, Sánchez-Palacios M, Hernández Medina E, Lorenzo Torrent R, Martínez Cuellar S, Villanueva Ortiz A. Pronóstico del paciente crítico según el sexo y la edad. Med Intensiva. 2009;33:161-5.

3. Garfield M, Ridley S, Kong A, Burns A, Blunt M, Gunning K. Seasonal variation in admission rates to intensive care units. Anaesthesia. 2001;56:1136-40.

4. Reinikainen $M$, Uusaro A, Ruokonen E, Niskanen M. Excess mortality in winter in Finnish intensive care. Acta Anaesthesiol Scand. 2006;50:706-11. 
5. Harrison DA, Lertsithichai P, Brady AR, Carpenter JR, Rowan K. Winter excess mortality in intensive care in the UK: An analysis of outcome adjusted for patient case mix and unit workload. Intensive Care Med. 2004;30:1900-7.

6. Keatinge WR, Coleshaw SRK, Cotter F, Mattock M, Murphy M, Chelliah R. Increases in platelet and red cell counts, blood viscosity, and arterial pressure during mild surface cooling: Factors in mortality from coronary and cerebral thrombosis in winter. BMJ. 1984;289:1405-8.

7. Millqvist E, Bengtsson U, Bake B. Occurrence of breathing problems induced by cold climate in asthmatics - a questionnaire survey. Eur Respir J. 1987;71:444-9.

8. Hodgin KE, Moss M. The epidemiology of sepsis. Current Pharmaceutical Design. 2008;14:1833-9.

9. Danai PA, Sinha S, Moss M, Haber MJ, Martin GS. Seasonal variation in the epidemiology of sepsis. Crit Care Med. 2007;35:410-15.

10. Reichert TA, Simonsen L, Sharma A, Pardo SA, Fedson DS, Miller $M A$. Influenza and the winter increase in mortality in the United States, 1959-1999. Am J Epidemiol. 2004;160:492-502.
11. Dowell SF, Whitney CG, Wright C, Rose Jr CE, Schuchat A. Seasonal patterns of invasive pneumococcal disease. Emerg Infect Dis. 2003;9:573-9.

12. Rennard SI, Higenbottam T. Exacerbation-free COPD: A goal too far?. Proc Am Thorac Soc. 2007;4:583-5.

13. Watson L, Turk F, James P, Holgate ST. Factors associated with mortality after an asthma admission: A national United Kingdom database analysis. Respir Med. 2007;101:1659-64.

14. Spencer FA, Goldberg RJ, Becker RC, Gore JM. Seasonal distribution of acute myocardial infarction in the second National Registry of Myocardial Infarction. J Am Coll Cardiol. 1998;31:1226-33.

15. Kysely J, Pokorna L, Kyncl J, Kriz B. Excess cardiovascular mortality associated with cold spells in the Czech Republic. BMC Public Health. 2009;9:19.

16. Lanska DJ, Hoffmann RG. Seasonal variation in stroke mortality rates. Neurology. 1999;52:984-90.

17. The Eurowinter Group. Cold exposure and winter mortality from ischaemic heart disease, cerebrovascular disease, respiratory disease, and all causes in warm and cold regions of Europe. Lancet. 1997;349:1341-6. 